\title{
Genetic mitochondrial glycine amidinotransferase protein aggregate formation triggers microparticle sensing and kidney failure
}

\author{
Shrikant R. Mulay, Hans-Joachim Anders \\ Division of Nephrology, Medizinische Klinik und Poliklinik IV, Klinikum der Universität München, LMU Munich, München, Germany \\ Correspondence to: Shrikant R. Mulay, PhD. Division of Nephrology, Medizinische Klinik und Poliklinik IV, Klinikum der Universität München, \\ LMU Munich, Schillerstr. 42, München 80336, Germany. Email: shrikant_ramesh.mulay@med.uni-muenchen.de. \\ Comment on: Reichold M, Klootwijk ED, Reinders J, et al. Glycine Amidinotransferase (GATM), Renal Fanconi Syndrome, and Kidney Failure. J Am \\ Soc Nephrol 2018;29:1849-58.
}

Submitted Jul 02, 2018. Accepted for publication Jul 10, 2018.

doi: 10.21037/atm.2018.07.09

View this article at: http://dx.doi.org/10.21037/atm.2018.07.09

Deposition of crystals or crystalline particles in various organs leads to diverse medical disorders (1). Misfolded or aggregated proteins can form crystalline or noncrystalline microparticles that activate similar molecular pathways of inflammation and cell death, for exampleAlzheimer's disease (amyloid- $\beta$ aggregates) (2), Parkinson's disease ( $\alpha$-synuclein misfolding) (3), or in monoclonal immunoglobulin-associated renal diseases $(4,5)$. A recent article by Reichold et al. published in The fournal of American Society of Nephrology demonstrate that monoallelic mutations in the gene encoding glycine amidinotransferase (GATM) promote linear aggregation of GATM protein inside the mitochondria of proximal tubular cells (6). These mitochondrial protein aggregate particles increased production of reactive oxygen species (ROS), followed by the NLRP3 inflammasome-dependent inflammation and cell death in kidneys of patients with renal Fanconi syndrome and chronic kidney disease (CKD).

In this study, the authors identified an autosomal dominant renal Fanconi syndrome in individuals from five extended families who developed glycosuria, hyperphosphosphaturia, generalized hyperaminoaciduria, low molecular weight proteinuria, and metabolic acidosis during their childhood that culminated in renal fibrosis and kidney failure with need for transplant or dialysis in adulthood. To understand the pathophysiology of this syndrome, the authors performed genome-wide linkage analysis, sequencing, and identified several heterozygous missense mutations in the GATM gene in all affected individuals from these extended families. Subsequently, histology analysis of kidney biopsies of affected individuals revealed the presence of GATM protein aggregates inside drastically enlarged mitochondria of proximal tubular cells. Furthermore, overexpression of mutant GATM proteins in the renal proximal tubular cell line LLC-PK1 lead to drastically enlarged mitochondria containing GATM-positive fibrillary aggregate particles similar to those observed in patients' biopsies whereas overexpression of wild-type GATM protein in LLC-PK1 did not show this abnormal mitochondrial morphology. In silico structural studies of GATM using X-ray crystallographic structures from the Protein Data Bank revealed the presence of an additional de novo interaction site on mutated GATM proteins, which support the formation of linear multimers of mutated GATM proteins. Interestingly, GATM-deficient mice did not display any aminoaciduria or glucosuria suggesting that the GATM protein aggregates, but not GATM protein per se, are responsible for the mitochondrial pathology leading to the signs and symptoms observed in patients.

Non-degradable protein deposits, as well as crystalline structures, have been reported to trigger the autoamplification loop of necroinflammation involving activation of NLRP3 inflammasome and regulated necrosis pathways (1-3,5,7-12). Consistent with these findings, the authors observed that the presence of mutant GATM protein aggregates in LLC-PK1 cells was associated with increased ROS production, enhanced expression of NLRP3 and interleukin 18 (IL-18), as well as increased rate of cell death. These results strongly suggest that mutant GATM protein aggregates share the same molecular pathway of necroinflammation which has been reported for other crystals or crystalline particles $(1,2,8-10,12)$. This implies 
that microparticles induce mitochondrial ROS production that ultimately triggers the formation of the NLRP3 inflammasome, a macromolecular complex consisting of NLRP3, the adaptor molecule ASC, and caspase-1 (13). Formation of this complex triggers enzymatic activation of caspase-1, which in turn cleaves the pro-forms of two important proinflammatory cytokines, i.e., pro-IL- $1 \alpha$ and pro-IL-1 $\beta(14,15)$. In addition, NLRP3 inflammasome activation has been linked to a particularly proinflammatory form of cell death, i.e., pyroptosis $(16,17)$. Pyroptosis is executed by gasdermin $\mathrm{D}$, a pore-forming protein, that integrates into the outer cell membrane leading to cell demise and release of intracellular molecules in the extracellular space (18). Such a release of intracellular molecules includes DAMPs (danger-associated molecular patterns) that can activate other danger-sensing receptors for sterile inflammation $(8,12)$. In this manner, intrinsic crystal-driven cell necrosis can trigger inflammation and further cell injury, i.e., necroinflammation $(8,12)$. Interestingly, LLC-PK1 cells expressing mutant GATM protein aggregates also exhibited increased fibronectin and smooth muscle actin mRNA levels suggesting a critical role of mitochondria in initiating pro-fibrotic signaling cascade. However, the involved mechanisms remain obscure.

The mitochondrial GATM enzyme catalyzes the biosynthesis of guanidinoacetic acid, the immediate precursor of creatine, which is regulate GATM expression in a negative feedback manner. Therefore, the authors suggest creatine supplementation as a pharmacological intervention to suppress endogenous production of mutated GATM protein. In fact, this treatment reduced production of both mRNA and protein of GTAM in wild-type mice. In addition, since mutated GTAM share molecular mechanism of necroinflammation, strategies targeted to combat crystalline particles induced-inflammation and regulated cell death could also serve as novel therapeutic options to halt the progression to kidney fibrosis and failure during CKD.

In summary, this study provides important evidence that mitochondrial aggregates of mutated GATM trigger the pathological events resulting in an autosomal dominant form of renal Fanconi syndrome, renal fibrosis and progressive organ failure. More significantly, this study emphasizes the importance of genetic diagnostic procedures to understand the underlying pathophysiology of CKD.

\section{Acknowledgements}

Funding: The Deutsche Forschungsgemeinschaft (MU
3906/1-1 to SR Mulay, and AN372/16-2 and 24-1 to HJ Anders) supported this work.

\section{Footnote}

Conflicts of Interest: The authors have no conflicts of interest to declare.

\section{References}

1. Mulay SR, Anders HJ. Crystallopathies. N Engl J Med 2016;374:2465-76.

2. Heneka MT, Kummer MP, Stutz A, et al. NLRP3 is activated in Alzheimer's disease and contributes to pathology in APP/PS1 mice. Nature 2013;493:674-8.

3. Codolo G, Plotegher N, Pozzobon T, et al. Triggering of inflammasome by aggregated alpha-synuclein, an inflammatory response in synucleinopathies. PLoS One 2013;8:e55375.

4. Sethi S, Rajkumar SV, D'Agati VD. The Complexity and Heterogeneity of Monoclonal Immunoglobulin-Associated Renal Diseases. J Am Soc Nephrol 2018;29:1810-23.

5. Mulay SR, Anders HJ. Crystal nephropathies: mechanisms of crystal-induced kidney injury. Nat Rev Nephrol 2017;13:226-40.

6. Reichold M, Klootwijk ED, Reinders J, et al. Glycine Amidinotransferase (GATM), Renal Fanconi Syndrome, and Kidney Failure. J Am Soc Nephrol 2018;29:1849-58.

7. Mulay SR, Desai J, Kumar SV, et al. Cytotoxicity of crystals involves RIPK3-MLKL-mediated necroptosis. Nat Commun 2016;7:10274.

8. Mulay SR, Linkermann A, Anders HJ. Necroinflammation in Kidney Disease. J Am Soc Nephrol 2016;27:27-39.

9. Mulay SR, Kulkarni OP, Rupanagudi KV, et al. Calcium oxalate crystals induce renal inflammation by NLRP3mediated IL-1beta secretion. J Clin Invest 2013;123:236-46.

10. Franklin BS, Mangan MS, Latz E. Crystal Formation in Inflammation. Annu Rev Immunol 2016;34:173-202.

11. Honarpisheh M, Foresto-Neto O, Desai J, et al. Phagocytosis of environmental or metabolic crystalline particles induces cytotoxicity by triggering necroptosis across a broad range of particle size and shape. Sci Rep 2017;7:15523.

12. Linkermann A, Stockwell BR, Krautwald S, et al. Regulated cell death and inflammation: an autoamplification loop causes organ failure. Nat Rev Immunol 2014;14:759-67.

13. Zhou R, Yazdi AS, Menu P, et al. A role for mitochondria 
in NLRP3 inflammasome activation. Nature 2011;469:221-5.

14. Gross O, Thomas CJ, Guarda G, et al. The inflammasome: an integrated view. Immunol Rev 2011;243:136-51.

15. Franchi L, Eigenbrod T, Munoz-Planillo R, et al. The inflammasome: a caspase-1-activation platform that regulates immune responses and disease pathogenesis. Nat Immunol 2009;10:241-7.

16. Bergsbaken T, Fink SL, Cookson BT. Pyroptosis:

Cite this article as: Mulay SR, Anders HJ. Genetic mitochondrial glycine amidinotransferase protein aggregate formation triggers microparticle sensing and kidney failure. Ann Transl Med 2018;6(16):315. doi: 10.21037/atm.2018.07.09 host cell death and inflammation. Nat Rev Microbiol 2009;7:99-109.

17. Gaidt MM, Hornung V. The NLRP3 Inflammasome Renders Cell Death Pro-inflammatory. J Mol Biol 2018;430:133-41.

18. Kayagaki N, Stowe IB, Lee BL, et al. Caspase-11 cleaves gasdermin D for non-canonical inflammasome signalling. Nature 2015;526:666-71. 